\title{
Estilo de gestão de produtores rurais
}

\author{
Management style of farmers
}

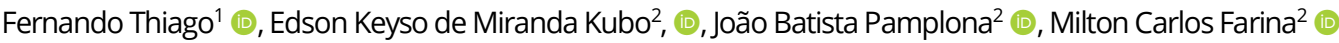 \\ 'Universidade Federal de Mato Grosso do Sul (UFMS), Campus do Pantanal, Corumbá (MS), Brasil. E-mail: fernando.t@ufms.br \\ ${ }^{2}$ Programa de Pós-graduação em Administração, Universidade Municipal de São Caetano do Sul (USCS), São Caetano do Sul \\ (SP), Brasil. E-mails: edson.kubo@prof.uscs.edu.br; joao.pamplona@prof.uscs.edu.br; milton.farina@prof.uscs.edu.br
}

Como citar: Thiago, F., Kubo, E. K. M., Pamplona, J. B., \& Farina, M. C. (2020). Estilo de gestão de produtores rurais. Revista de Economia e Sociologia Rural, 58(2), e188254. https://doi.org/10.1590/1806-9479.2020.188254

Resumo: O objetivo desta pesquisa foi caracterizar o Estilo de Gestão de produtores rurais no Brasil. Tratou-se Estilo de Gestão como a manifestação das características de se administrar a interação entre as pessoas e entre pessoas e a estrutura, de um ou coletivo de gestores de uma determinada organização. Para analisar o Estilo de Gestão dos produtores rurais, foram considerados os fatores Liderança, Decisão, Comunicação, Controle, Planejamento e Motivação. A abordagem mista subsidiou a metodologia da pesquisa, tendo a análise quantitativa a partir da técnica de levantamento (survey) e análise qualitativa utilizando a técnica de grupo focal, sendo que, para cada técnica de coleta de dados, foram utilizadas amostras diferentes. A amostra dos participantes do levantamento consistiu em 101 gestores de organizações produtoras rurais brasileiras. Participaram da reunião do grupo focal 5 profissionais com experiência e conhecimento na área de gestão de organizações rurais. Os resultados mostraram que os produtores rurais possuem um Estilo de Gestão caracterizado pela forte presença de atividades referentes à liderança, decisão, controle, motivação e tecnologia e menor relevância nos aspectos planejamento e comunicação.

Palavras-chave: estilo de gestão, sistema de gestão, modelo de gestão, agropecuaristas.

\begin{abstract}
The objective of this research was to characterize the Management Style of farmers in Brazil. Management Style was treated as the manifestation of the characteristics of managing the interaction between people, and between people and the structure of one or collective of managers of a given organization. In order to analyze the Management Style of the farmers were considered the factors as Leadership, Decision, Communication, Control, Planning and Motivation. The mixed approach subsidized the research methodology, taking the quantitative analysis from the survey technique and qualitative analysis using the focus group technique, and for each technique of data collection, different samples were used. The sample of survey participants consisted of 101 managers from Brazilian farm organizations. For the focus group meeting, 5 professionals with experience and knowledge in the area of management of rural organizations participated. The results showed that farmers have a Management Style characterized by the strong presence of activities related to leadership, decision, control, motivation and technology, and less relevance in the planning and communication aspects.
\end{abstract}

Keywords: management style, management systens, management model, brazilian farmers.

\section{Introdução}

O Brasil é uma das dez maiores economias do mundo apresentando um rápido e significante desenvolvimento econômico nos últimos 20 anos. Este fator pode ser comprovado observando o Produto Interno Bruto (PIB) que representou quase US\$2 trilhões em 2015. Com a $5^{a}$ maior população do mundo (206 milhões) e $5^{a}$ área territorial (852 milhões de hectares), o Brasil tem um clima favorável que contribui para ser um dos players globais da agropecuária, fornecendo alimentos para o mercado doméstico e internacional (Hubbard et al., 2017). 
Neste período a agricultura brasileira deu um salto significativo, não apenas em termos de grande aumento de produção e produtividade de suas principais commodities, tais como café, soja, açúcar, carne bovina, milho, aves e carne de porco, mas também nas condições sociais e qualidade de vida, especialmente em termos de renda, saúde, educação e serviços de seus habitantes rurais. Por exemplo, o número de pessoas na pobreza caiu de 50 milhões em 1996 para menos de 3 milhões em 2015 (Bojanic, 2017).

Embora a infraestrutura produtiva ainda seja considerada um grande gargalo, especialmente de logística, os produtores rurais puderam usufruir de uma melhoria significativa, por exemplo, nos processos de irrigação, armazenamento, estradas e vias navegáveis. Tudo isso contribuiu para o Brasil se tornar um dos líderes mundiais mais importantes na produção agrícola e no comércio agrícola internacional (Bojanic, 2017).

O Brasil é o segundo maior exportador agrícola do mundo. Este desempenho impressionante pode ser atribuído a uma combinação de fatores, tais como melhorias na produtividade, adoção de novas tecnologias e os ajustes estruturais após grandes reformas econômicas nos anos 1980 e 1990 (Hubbard et al., 2017). Estas reformas econômicas estabeleceram um ambiente mais competitivo, facilitaram a consolidação da terra e a adoção de novas tecnologias pelas grandes explorações comerciais, levando a um uso mais eficiente dos fatores de produção agrícola, em particular a terra e o trabalho (Moreddu et al., 2017).

$\mathrm{O}$ ano 2000 e os anos seguintes foram marcados pela grande recuperação do setor pós-crise econômica originada após a abertura dos mercados nos anos 1990, retomando com a produção de commodities de baixo valor agregado voltadas principalmente para o mercado externo. Neste período, o Estado passou de regulador de preços mínimos combinados para investimentos na cadeia produtiva e avanço no crédito rural (Silva, 1996; Virgílio, 2017). Além disso, destaca-se a forte aplicação e desenvolvimento de tecnologias avançadas e a internacionalização da cadeia produtiva (Virgílio, 2017).

Outro fator é o investimento em pesquisa e desenvolvimento, serviços de extensão agrícola, especialmente os conduzidos pela Empresa Brasileira de Pesquisa Agropecuária (EMBRAPA), e infraestrutura também foram cruciais. Apesar desse sucesso, o setor ainda enfrenta uma série de desafios pertinentes à competitividade, especialmente no tocante às estruturas logísticas, acesso a recursos de capital e mão de obra qualificada (Bojanic, 2017; Hubbard et al., 2017; Moreddu et al., 2017).

Os ganhos de produtividade levaram o Brasil de um grande importador de alimentos, sujeito a grandes crises de abastecimento, a um líder global de exportação de uma diversidade de produtos agrícolas. O período de 1975 a 2014 apresentou um aumento de produção; $92 \%$ do aumento foi devido ao aumento de produtividade e $8 \%$ ao crescimento do uso de insumos, de terra, mão de obra e capital (Gasques, 2017).

Como resultado, a produtividade atingiu uma média de crescimento anual de 3,5\% em relação a 1975-2012 (Gasques, 2017), mesmo diante de um panorama mundial em que países exportadores de produtos agropecuários têm apresentado redução ou mesmo estagnação de suas taxas de produtividade. A agricultura brasileira continua registrando um dos maiores desempenhos de produtividade no mundo (Moreddu et al., 2017).

As principais causas do aumento da produtividade no Brasil, além das mudanças políticas e desenvolvimento econômico, especialmente após a década de 1990, são dispensadas aos fatores tecnológicos tais como: as safrinhas; cultivares genéticas de alta resistência a doenças; cobertura de palha para manter níveis de matéria orgânica no solo (Gasques, 2017).

Por outro lado, os produtos agropecuários têm se tornado também fonte de matériaprima para indústrias de outras naturezas como energia, como o caso dos biocombustíveis; polímeros e produtos químicos de base biológica e fibras sintéticas; produtos farmacêuticos ou de saúde, como os alimentos funcionais, hormônios de crescimento e materiais cirúrgicos (King et al., 2010).

Tais possibilidades na utilização desses produtos proporcionam ao setor diferentes desafios estratégicos e competitivos. Além disso, toda a discussão sobre as mudanças climáticas pode ainda prover novos remodelamentos nas formas de gestão, adequação e mudanças nos posicionamentos estratégicos (King et al., 2010). 
Diante disso, as empresas de produção rural vêm se tornando cada vez mais flexíveis e complexas, cujos gestores são impelidos ao desafio da implantação dos modelos de gestão que atendam às novas demandas produtivas e socioambientais (King et al., 2010).

Neste aspecto, valores, crenças, missão e práticas de gestão mantêm um estilo que pode gerar vantagem competitiva para a organização atuar neste cenário (Petry \& Nascimento, 2009; Rafiq \& Chishti, 2011; Campos et al., 2015; Oliveira et al., 2016).

Diante da contextualização apresentada, esta pesquisa se propõe a articular a literatura envolvida, resgatando o construto em sua ideia inicial, reformulando e discutindo sua forma de mensuração dentro do contexto da gestão de empresas produtoras rurais.

Para tanto, as organizações agropecuaristas brasileiras foram utilizadas como objeto de pesquisa para avaliação de seu estilo de gestão, por contemplarem um dos mais representativos setores brasileiro. Justifica-se a escolha pela representatividade econômica e social do setor que atualmente movimenta US\$ 88 bilhões (Brasil, 2016), também por não constar outra pesquisa que avalie o estilo de gestão deste setor brasileiro, conforme verificado em banco de periódicos, teses e dissertações da CAPES e nas bases de pesquisas do SPELL, EBSCO e ProQuest.

\section{Estilo de Gestão}

Os principais temas abordados nesta investigação são os pertinentes às pesquisas sobre Estilo de Gestão, dentro de suas diversas terminologias, conceitos e instrumentos.

As pesquisas que se intitulam ou apresentam como palavras-chave "Estilo de Gestão", "Sistema de Gestão" e "Modelos de Gestão", ou seus respectivos termos na língua inglesa "Management Style", "Managerial Style", "Management System" ou "Management Model", trazem, em suas entrelinhas, assuntos pertinentes à forma ou modos de operar a gestão de organizações por seus gestores. Algumas pesquisas utilizam de maneira intercambiável o construto Estilo de Gestão como Liderança, baseando-se principalmente nas teorias referentes aos traços de liderança dos gestores, como na pesquisa de Lindell \& Arvonen (1996), Mukherji et al. (2009), Shadare (2011), ou gestão de sistema de informações como em lizuka et al. (2014), ou mesmo em processos de tomada de decisão como em Poon et al. (2005).

Conceitualmente o construto Estilo de Gestão foi definido como um conjunto de características recorrentes que estão associadas com o processo decisório da empresa ou gerentes individuais (Tull \& Albaum, 1971).

Para Guerreiro (1989, p. 62), os estilos de gestão são "as imagens intelectuais sobre as quais se desenvolve o conhecimento obtido de um trabalho explícito ou não, de seleção dos elementos relevantes da porção da realidade em análise".

Assim, os estilos de gestão, que também se configuram como um tipo de modelo de gestão, representam as características organizacionais refletidas na forma que o(s) gestor(es) age(m) e atinge $(\mathrm{m})$ seus objetivos, com características próprias como: centralizador/ descentralizador, níveis de comprometimento e responsabilidade, delegação e empowerment (Pereira \& Santos, 2001; Petry \& Nascimento, 2009; Gomes et al., 2013).

Observa-se a amplitude de avaliação no que tange ao objeto de investigação do Estilo de Gestão, sendo um a organização (Tull \& Albaum, 1971; Pereira \& Santos, 2001; Petry \& Nascimento, 2009; Gomes et al., 2013) ou gestores (Tull \& Albaum, 1971; Abramson et al., 1993; Pereira \& Santos, 2001; Petry \& Nascimento, 2009; Gomes et al., 2013).

Outro aspecto observado é quanto às saídas para se identificar o estilo de gestão. Para os autores Pereira \& Santos (2001), Petry \& Nascimento (2009) e Gomes et al. (2013), a base para identificar o estilo de gestão está no planejamento; para Tull \& Albaum (1971), está no processo decisório, e para Abramson et al. (1993), está na forma de resolução de problemas.

Com o objetivo de continuar o debate sobre o conceito de Estilo de Gestão para esta pesquisa e baseado em Likert (1967), Likert \& Likert (1976), Tull \& Albaum (1971), Guerreiro (1989), Abramson et al. (1993), Culpan \& Kucukemiroglu (1993), Pereira \& Santos (2001), Blanco Jiménez et al. (2009), Petry \& Nascimento (2009), Rafiq \& Chishti (2011), Gomes et al. (2013) e Gupta et al. (2014) definiu-se: Estilo de Gestão como a manifestação das características de se administrar a interação entre as pessoas e entre pessoas e a estrutura, 
de um ou coletivo de gestores de uma determinada organização, utilizando-se dos seus processos cognitivos que refletem todas suas atividades.

Esta definição aborda dois aspectos: o primeiro relacionado à gestão da interação das pessoas nos exercícios de suas atribuições e à forma como elas utilizam a estrutura e materiais necessários para atendimento a exigências de seus cargos; o segundo aspecto retrata a forma como os gestores utilizam seus conhecimentos prévios e os conhecimentos adquiridos durante seu período de gestão, além de considerar também a forma como tais conhecimentos são adquiridos.

Contudo, Zeffane (1994) verificou que estudos sobre Estilo de Gestão têm se concentrado na noção de Estilo de Liderança em um nível individual. Porém essa noção mais ampla da organização é passível de verificação quando direcionada para os aspectos da Cultura Corporativa.

Esta evidência permitiu revisitar os conceitos e escalas, verificando uma certa ligação destes com o nível individual, como visto nos trabalhos de Tull \& Albaum (1971), Abramson et al. (1993), Pereira \& Santos (2001), Petry \& Nascimento (2009) e Gomes et al. (2013). Assim, propor a inclusão de dimensões e itens da Cultura Organizacional juntamente com as dimensões envolvidas para mensurar o Estilo de Gestão pode inviabilizar a execução da pesquisa, apresentando baixos níveis de parcimônia.

Mesmo que não utilizando aspectos relacionados à cultura organizacional, mas empregando dimensões de avaliação com sentenças do instrumento para uma análise não apenas abordando o nível individual, mas também organizacional, conforme discutido por Zeffane (1994), possibilita verificar empiricamente uma alternativa para a lacuna observada na literatura.

Diante disso, foram revisitadas as teorias de cada uma destas dimensões utilizadas, as quais estão representadas no Quadro 1.

Quadro 1. Aspectos teóricos utilizados nas dimensões.

\begin{tabular}{|c|c|}
\hline Dimensão e aspecto teórico & Apoio teórico \\
\hline $\begin{array}{l}\text { Liderança: Liderança } \\
\text { Transformacional e transacional }\end{array}$ & $\begin{array}{l}\text { Avolio et al. (1991), Bass \& Avolio (1997) e Ghasabeh et al. } \\
\qquad(2015) .\end{array}$ \\
\hline $\begin{array}{l}\text { Decisão: Racionalidade limitada, } \\
\text { Garbage Can. }\end{array}$ & $\begin{array}{c}\text { Simon (1945), Eisenhardt \& Zbaracki (1992), Robbins \& } \\
\text { Decenzo (2004), Huang (2009), Child et al. (2010), Lomi et al. } \\
\text { (2012), Graffin et al. (2013). }\end{array}$ \\
\hline $\begin{array}{l}\text { Comunicação: Meios de } \\
\text { Comunicação, Qualidade da } \\
\text { comunicação e Interação social. }\end{array}$ & $\begin{array}{c}\text { Hall (2003), Cardoso (2006), Sousa (2006), Serra (2007), } \\
\text { Marchiori (2009), Maguire \& Hardy (2009), Souza (2010), } \\
\text { Franco \& Leão (2013), Spaho (2013), Mariño-Arévalo (2014) e } \\
\text { Alhassan et al. (2014). }\end{array}$ \\
\hline $\begin{array}{l}\text { Controle: Controle gerencial, } \\
\text { Sistema de diagnóstico, Controle } \\
\text { estratégico, Interatividade dos } \\
\text { sistemas, Sistema de crenças e } \\
\text { Sistema de restrições. }\end{array}$ & $\begin{array}{c}\text { Simons (1995), Merchant \& Van Der Stede (2007), Grabner \& } \\
\text { Moers (2013) e Hared et al. (2013). }\end{array}$ \\
\hline $\begin{array}{l}\text { Planejamento: Estabelecimento da } \\
\text { Missão, Valores e Visão, } \\
\text { Diagnóstico, Definição dos } \\
\text { objetivos e metas, Definição do } \\
\text { planejamento e Implementação do } \\
\text { planejamento. }\end{array}$ & $\begin{array}{l}\text { Mintzberg (2008), Fischmann \& Almeida (2009), } \\
\text { Guimarães et al. (2010), Poister (2010), Pereira (2011), Souza } \\
\text { \& Marinho (2014) e Oliveira (2014). }\end{array}$ \\
\hline Motivação: extrínseca e intrínseca. & Deci et al. (1996), Cokley (2000), Pinder (2008) e Ertas (2015). \\
\hline
\end{tabular}

Fonte: elaborado pelos autores.

A formação dimensional do construto considera-se uma das questões sem consenso nas pesquisas sobre Estilo de Gestão, sendo as mais representativas: Liderança, Decisão, 
Comunicação, Controle, Planejamento e Motivação (Likert, 1967; Likert \& Likert, 1976; Morris \& Pavett, 1992; Culpan \& Kucukemiroglu, 1993; Beechler \& Yang, 1994; Glunk et al., 1996; Pheng \& Leong, 2001; Blanco Jiménez et al., 2009; Petry \& Nascimento, 2009; Gomes et al., 2013; Gupta et al., 2014), estas dimensões foram utilizadas para avaliação desta pesquisa e são possíveis de serem identificadas nas organizações participantes da pesquisa: empresas agropecuaristas.

\section{Organizações agropecuaristas no contexto brasileiro}

No Brasil, o agronegócio começou a se desenvolver na década de 1950 com a utilização de máquinas a vapor nas usinas de cana no nordeste e utilização de máquinas no arroz e trigo no sul do país. Mas foi a partir da década de 1970, com a política de modernização da agricultura, que começou a se desenvolver mais fortemente e ser competitiva no mundo, aparecendo, neste período, nomenclaturas como "empresas rurais" e "empresários rurais". A partir da década de 1980, termos como "agroindústria", "agronegócio" e "complexos industriais" surgem num contexto em que profissionalização e industrialização da agricultura passam a ser representativos nos contextos econômico e acadêmico, sendo o termo "agronegócio" mais frequente a partir do boom das exportações (Heredia et al., 2010).

A partir de então, as pressões econômicas de ambientes de competitividade fizeram com que as empresas rurais se especializassem cada vez mais, apresentando uma revolução tecnológica para melhorar a produção, em especial a indústria química e motomecânica. Neste período, processos como terceirização de produtos, antes produzidos dentro da propriedade rural, passaram a ser realizados por outras empresas, tais como: fertilizantes, defensivos, vacinas, máquinas e implementos, rações, genética, entre outros, os chamados elementos "antes da porteira", como também a terceirização da comercialização, distribuição e transporte, denominados de atividades "depois da porteira" (Goodman et al., 2008).

Essa especialização "dentro da porteira", ou seja, todas as atividades realizadas na propriedade rural, foco do negócio, permitiu que ocorresse um aumento na produtividade ano a ano pelas empresas agropecuaristas, sendo um dos setores mais representativos em termos econômicos no Brasil, uma vez que responde por $22,74 \%$ do PIB nacional, com crescimento acelerado, sendo que em 2013, segundo o CEPEA/USP (Centro de Estudos Avançados em Economia Aplicada, 2013), obteve um crescimento 4,46\%.

Em termos mundiais, o Brasil está em primeiro lugar em produção de açúcar, café e suco de laranja e em segundo lugar em soja, carne bovina, tabaco e cana-de-açúcar (etanol). É o país do globo que mais exporta açúcar, café, suco de laranja, carne bovina, tabaco, cana-de-açúcar (etanol) e aves; em $2^{\circ}, 3^{\circ}$ e $4^{\circ}$ lugar estão respectivamente: soja, milho e carne suína no mundo (Brasil, 2010). O superávit da balança comercial do agronegócio está em US\$83 bilhões, sendo significativo quando comparado com o superávit geral brasileiro de US\$17 bilhões. Diante disso, observa-se que o agronegócio sustenta o status de superávit da balança comercial brasileira (Brasil, 2016).

Segundo o Instituto Matogrossense de Economia Agropecuária (Instituto Matogrossense de Economia e Agropecuária, 2014a), as vantagens competitivas do setor estão ligadas à produção em escala, tecnologia empregada, empreendedores e portfólio. As desvantagens são: logística, burocracia, tributos e insegurança jurídica; o fator "mercado interno" é posicionado como intermediário entre vantajoso e desvantajoso.

Em favor deste quadro, os estilos de gestão nas organizações agropecuaristas tornam-se cada vez mais importantes no sentido de desenvolver, reter e promover trabalho com eficiência e eficácia diante dos desafios presentes, em soluções inovadoras para melhorar as desvantagens competitivas, como também para os desafios futuros principalmente com o aumento da população mundial e o fortalecimento econômico de países em desenvolvimento, gerando maior demanda de produtos diversos, especialmente os alimentícios e energéticos.

Cabe esclarecer que, segundo Davis \& Goldeberg (1957), agronegócio trata-se de um conjunto de todas as operações, transações, atividades produtivas, desde a produção dos insumos, passando pela produção, armazenamento, até o processamento e distribuição das commodities e os produtos derivados delas, englobando todas as organizações que afetam o ciclo produtivo e de distribuição. 
A agropecuária compreende a produção no campo como: cultivo de soja; criação de gado; aquicultura, etc., produzidos diretamente de unidades rurais, fazendas, sítios e chácaras (Instituto Matogrossense de Economia e Agropecuária, 2014b), sendo um dos elos do agronegócio.

Para fins de definição das organizações participantes, utilizou-se a Classificação Nacional de Atividades Econômicas (CNAE), na qual a agropecuária compreende atividades para o cultivo de lavouras e a criação e produção animal e possui suas subdivisões (Instituto Brasileiro de Geografia e Estatística, 2010).

Assim, esta pesquisa foi realizada nas organizações de atividades agropecuárias brasileiras e a escolha destas organizações se deve principalmente por sua representatividade econômica no cenário brasileiro e mundial, mas também devido a suas características permitirem responder à questão de pesquisa. Neste aspecto, observa-se que estas organizações possuem elementos organizacionais que podem ser evidenciados por meio dos fatores que compõem o Estilo de Gestão - Liderança, Decisão, Comunicação, Planejamento, Controle e Motivação - buscando compreender os aspectos tanto individuais dos seus gestores como também os organizacionais.

\section{Procedimentos Metodológicos}

Trata-se de uma pesquisa mista utilizando a técnica de levantamento (quantitativo) e focus group (qualitativo). O levantamento de campo ou survey é caracterizado pelo questionamento direto a uma amostra de pessoas sobre o comportamento ou opiniões que se pretende conhecer (Gil, 2008; Fowler Junior, 2011). Segundo Gil (2008), os procedimentos de análise dos dados característicos desse tipo de técnica são de ordem quantitativa, pelas quais pode-se chegar a conclusões sobre o fenômeno estudado.

A amostra consistiu de 111 gestores agropecuaristas brasileiros, sendo que 10 foram entrevistados para a avaliação do instrumento-piloto e 101 para avaliação do Estilo de Gestão, localizados em 11 Estados Brasileiros, sendo eles: Rio Grande do Sul, Santa Catarina, Paraná, São Paulo, Minas Gerais, Mato Grosso do Sul, Mato Grosso, Goiás, Rondônia, Roraima e Pará.

Para esta análise, selecionamos dados pertinentes ao gestor ou à propriedade rural, com características métricas e nominais.

Os participantes foram selecionados por amostragem não probabilística por acessibilidade. Segundo Costa Neto (1977), nesta técnica de amostragem, mesmo que se possa obter os dados de toda a população, retira-se apenas a amostra dos participantes que estejam acessíveis para responder (Costa Neto, 1977).

Em relação ao instrumento de coleta de dados para o levantamento, a elaboração e sua sequente validação passaram pelas etapas de (1) avaliação semântica do instrumento com a aplicação de pré-teste, realizado pelo teste-piloto; e (2) procedimentos para validação empírica com aplicação do instrumento e posterior análise estatística (Dantas, 2007; Pelegrino, 2009; Pasquali, 2010).

Para validação empírica, foi utilizada a técnica de Análise Fatorial Exploratória Unidimensional, com apoio do software SPSS (v. 21), a qual permite extrair os fatores que compõem um determinado construto. De acordo com Hair Jr. et al. (2010), esta técnica tem como objetivo desenhar uma forma de resumir informações presentes nas variáveis de um determinado fenômeno, em um agrupamento menor de novos fatores ou dimensões com a menor perda possível de informações.

Embora este tipo de teste estatístico seja mais bem aplicado aos construtos em formação, com dados empíricos e teorias que não permitam o seu grupamento a partir de parâmetros conhecidos, a sua utilização de forma unidimensional, ou seja, aplicada a cada fator, permite verificar o quão bem as variáveis se ajustam aos seus respectivos fatores.

Segundo Vitória et al. (2006, p. 5), "o raciocínio de base é simples: se um teste é unidimensional, quando submetido à análise fatorial ou à análise em componentes principais emergirá um único fator". Considerando que se apenas um fator for responsável pelas variáveis do fenômeno, logo este fator explicaria toda a variância observada. Contudo, neste caso de pesquisa, o fenômeno observado é de pertinência das ciências sociais, sendo impossível a obtenção de um nível de explicação de 100\%, mas possibilita checar o grau de 
unidimensionalidade do fator (Vitória et al., 2006). Esse grau de unidimensionalidade possibilita analisar o quanto as variáveis se ajustam ao fator.

Diante disso, foram realizados os testes de Kaiser-Meyer-Olkin (KMO) e de Esfericidade de Bartlett, extraídos pelos métodos de principal componente. Além de verificar a extração, foi realizado o teste de Measure of Sampling Adequacy (MSA) para verificação da aderência de cada variável. Como a análise foi realizada por fator, não se avaliou o teste para decisão dos fatores extraídos, normalmente realizado pela técnica de rotação Varimax com normalização de Kaiser (Hair Junior et al., 2010).

A avaliação do Estilo de Gestão foi realizada por duas formas de análise: a primeira é a análise dos dados obtidos pela Escala de Avaliação do Estilo de Gestão; a segunda é obtida por meio de grupo focal de discussão dos resultados obtidos na primeira forma de avaliação.

As variáveis contidas nos fatores foram medidas e analisadas pelo score fatorial médio, sendo que, quanto maior for o valor que o participante perceber da variável respondida, maior será a relevância dada ao fator analisado. Além disso, as medidas de variâncias, como o índice de desvios médios ou desvio padrão das respostas, permitem a análise de concordância entre os participantes da pesquisa, verificando a existência de consenso intragrupo (Severino, 2010).

A segunda etapa consistiu na discussão dos dados obtidos por um grupo focal, utilizando a abordagem qualitativa e tipo descritiva. De acordo com Gil (2008), o grupo focal consiste em uma técnica de entrevista em grupo de forma que o pesquisador figura como moderador de um determinado número de participantes que varia de 5 a 12 pessoas (Gil, 2008).

A utilização de coleta e análise de dados obtidos por meio de grupo focal e a comparação dos resultados obtidos no levantamento (survey) se caracteriza como uma estratégia ímpar, permitindo aprofundamento empírico do fenômeno estudado, como também comparar métodos de pesquisa, como observado nos trabalhos de De Koning et al. (2015), Porpino et al. (2015), Armstrong et al. (2016), Hartono \& Sobari (2016), Lam (2016), Perreira \& Berta (2016), Schumann et al. (2016) e Srivastava \& Banerjee (2016), ou para complementação de outros tipos de técnicas de pesquisa como entrevistas individuais (Dufour-Poirier \& Laroche, 2015; Wanyonyi et al., 2015; Zakrison et al., 2015; Banks et al., 2016; Kistruck et al., 2016, MartínezAires et al., 2016), entrevistas e observação (Rivers et al., 2015; Srivastava \& Banerjee, 2016),

No total, realizou-se um grupo focal de 5 participantes, da carreira docente da educação superior, pesquisadores caracterizados pela experiência e conhecimentos na área de gestão de organizações agropecuaristas. A pauta da reunião consistiu na apresentação dos resultados coletados pela Escala do Estilo de Gestão.

Após a execução da reunião, as conversas foram transcritas e analisadas por meio da técnica de análise de conteúdo descrita por Bardin (1977), consistindo de 3 etapas: préanálise, exploração do material e tratamentos dos dados e interpretação. Como apoio foi utilizado o software Atlas TI. Esta técnica permite identificar temas emergentes descritos pelos participantes e codificados em categorias de análises.

\section{Resultados e Discussões}

A maioria dos participantes da pesquisa são do sexo masculino $(84,1 \%)$, mais da metade $(53,5 \%)$ está nos Estados de Mato Grosso, Rio Grande do Sul e Minas Gerais. A maior parte dos participantes $(66,4 \%)$ possui curso superior e pós-graduação. São casados(as) ou vivem com companheiro(a) $80,2 \%$. As ocupações que mais se destacaram na amostra foram as de proprietários e gestores $(56,4 \%)$. A idade dos participantes está entre 31 e 38 anos; em relação ao tempo de serviço, a maioria dos participantes atua há 25 anos na empresa; a média e mediana estão praticamente em 15 anos, com baixo desvio padrão relativizando que os participantes do conjunto de dados estão entre 15 e 25 anos.

Em relação às características das propriedades, a maioria $(83,1 \%)$ está vinculada a algum tipo de órgão sindical ou associativo. As produções mais recorrentes nas empresas rurais são derivadas das atividades de agricultura $(61,8 \%)$ e pecuária $(34,3 \%)$. A maioria das organizações possui de 2 a 3 funcionários. Seu tempo de existência é, em média, 35 anos e possui, em média, 5115,83 hectares de área. 


\subsection{Análise dos dados da Escala de Estilo de Gestão}

Os dados coletados pela Escala de Estilo de Gestão permitiram a expressão das percepções sobre as atividades desempenhadas pelos gestores de propriedades rurais. As medidas de tendências centrais, desvio padrão e amplitude destas emissões estão expostas na Tabela 1.

Tabela 1. Scores do Estilo de Gestão por fator.

\begin{tabular}{|c|c|c|c|c|c|c|c|}
\hline \multirow[b]{2}{*}{ Medidas } & \multicolumn{6}{|c|}{ Dimensões } & \multirow{2}{*}{$\begin{array}{l}\text { Estilo } \\
\text { de } \\
\text { Gestão }\end{array}$} \\
\hline & Liderança & Decisão & Comunicação & Controle & $\begin{array}{l}\text { Planeja- } \\
\text { mento }\end{array}$ & Motivação & \\
\hline Média & 4,24 & 3,90 & 3,31 & 3,73 & 3,64 & 3,83 & 3,74 \\
\hline Mediana & 4 & 4 & 3 & 4 & 4 & 4 & 3,68 \\
\hline $\begin{array}{l}\text { Desvio } \\
\text { padrão }\end{array}$ & 0,71 & 0,87 & 1,13 & 1,03 & 1,24 & 0,75 & 0,82 \\
\hline Amplitude & 3 & 4 & 4,67 & 3,50 & 5 & 3 & 3,45 \\
\hline
\end{tabular}

Fonte: Coleta de campo (2017).

Os scores de cada dimensão apresentados foram calculados a partir da soma dos valores das percepções emitidas pelos participantes de cada variável e divididos pela quantidade de variáveis, formando os valores de cada dimensão.

A primeira dimensão verificada foi a liderança. As sentenças utilizadas avaliaram o quanto o líder possui características da liderança transformacional, sendo os scores próximos a 4 e 5, caracterizando os líderes como transformacionais, os scores próximos a 2 e 3, caracterizando como transacionais e os scores 1 e 2, laissez-faire. No caso observado, os líderes se consideram transformacionais, com opiniões muito próximas, apoiados pelo baixo desvio padrão.

Com base em Avolio et al. (1991), Bass \& Avolio (1997) e Ghasabeh et al. (2015), os líderes desenvolvem uma visão compartilhada entre eles, que proporciona melhor relação com seus colaboradores. Passam a identificar as necessidades dos funcionários, especialmente as relacionadas à capacitação dos funcionários, provendo um clima de aprendizagem na organização. Além disso, concentram-se no atendimento das expectativas das pessoas.

Em relação aos procedimentos pertinentes à tomada de decisão, as pontuações emitidas correspondem à presença de característica de tomada de decisão programada e participativa. Considerando a escala de mensuração, nas organizações analisadas, a programação e a participação dos funcionários são utilizadas em cerca de $80 \%$ (ponto 4 da escala) das vezes em que as decisões são tomadas.

As sentenças utilizadas para a formação desta dimensão consistem na busca de informações para fomentar a decisão conforme abordado por Ramos et al. (2015) e na participação de funcionários e demais envolvidos na decisão, de acordo com Robbins \& Decenzo (2004).

A dimensão Comunicação foi a que obteve a menor média e mediana (3,1 e 3) e o segundo maior desvio padrão e amplitude. Estes dados mostram que esta área de gestão das propriedades rurais merece mais atenção de seus gestores e suas organizações associativas. Considerando seu alto desvio padrão e sua amplitude, quase do tamanho da escala $(4,6)$, esclarece que os processos de comunicação organizacional não possuem práticas semelhantes nas organizações participantes, considerando os aspectos avaliados.

Neste ponto, as sentenças utilizadas na verificação desta dimensão consideraram a existência de comunicações formais (Sousa, 2006; Cardoso, 2006; Serra, 2007), a qualidade das informações emitidas com atributos de clareza e fácil entendimento (Sousa, 2006; Marchiori, 2009; Franco \& Leão, 2013; Spaho, 2013; Mariño-Arévalo, 2014; Alhassan et al., 2014) e a interação social nos processos comunicativos, permitindo que todos avaliem e discutam as comunicações recebidas e as formulações das comunicações enviadas (Hall, 
2003; Maguire \& Hardy, 2009; Souza, 2010; Franco \& Leão, 2013; Spaho, 2013; Mariño-Arévalo, 2014; Alhassan et al., 2014).

Em relação à dimensão Controle, a teoria observada nas sentenças é pertinente à utilização de indicadores externos, tais como demanda pela produção, valor do dólar, fatores climáticos, etc., conforme discutido por Merchant \& Van Der Stede (2007). A tipologia de Simons (1995) foi relevante para propor base analítica baseada em sistemas de controle por crença, utilizando indicadores como os valores organizacionais, sistema de diagnóstico e restrições. Além destas, as interações dos diversos sistemas, conforme discutido por Grabner \& Moers (2013) e Hared et al. (2013), foi utilizada.

Assim, os participantes avaliaram que as atividades de controle estão entre o melhor nível e o intermediário, apresentando que as organizações utilizam instrumentos e indicadores para controle organizacional em aproximadamente $80 \%$ das vezes, como observado na média 3,7 e mediana 4 . O desvio padrão desta dimensão também se apresenta como baixo $(1,09)$, mostrando uma certa concordância entre os participantes.

Em relação ao Planejamento, os indicadores formativos da dimensão apontaram uma avaliação entre a melhor pontuação possível e o nível regular. A estatística média obteve a pontuação 3,64 e a mediana obteve pontuação 4.

O desvio padrão e a amplitude de respostas indicaram que os participantes não emitiram suas percepções da mesma maneira, mostrando pouco consenso. Apoiando esta afirmação, dentre as dimensões analisadas, o Planejamento apresentou maior amplitude (5), ou seja, obtiveram-se respostas com valores entre 1 e 5.

Ainda em relação às avaliações de medidas centrais da dimensão Planejamento, embora não seja a avaliação mais baixa, requer atenção, uma vez que é possível se avançar mais nas questões relativas aos processos de planejamento, ora pela avaliação média e mediana próxima a 4, ora pela falta de unicidade entre as avaliações dos participantes.

A composição da dimensão tratou dos momentos para a efetivação do planejamento, sendo eles: (1) Estabelecimento da Missão, Valores e Visão; (2) Diagnóstico; (3) Definição dos objetivos e metas; e (4) Definição do planejamento, os quais foram utilizados para elaboração das sentenças deste construto (Mintzberg, 2008; Fischmann \& Almeida, 2009; Cummings \& Daellenbach, 2009; Souza \& Marinho, 2014), como também considerou-se o momento de implementação do planejamento (Vaitsman et al., 2006; Guimarães et al., 2010; Pereira, 2011).

Conforme a literatura observada, esses são passos essenciais para delimitar um planejamento organizacional, como sugerido na hipótese de correlação desta dimensão com os dados organizacionais como produção, área da propriedade e número de funcionários.

O último fator avaliado foi a Motivação. Trata-se da dimensão com maior número de variáveis, seis no total. Os valores observados mostram a média e mediana próximas ao valor 4 ocorrendo em $80 \%$ das atividades que utilizam essa área da gestão. Assim como na dimensão liderança, ocorreu uma percepção muito semelhante dos participantes quando verificado o teste estatístico do desvio padrão $(0,75)$ e a amplitude das respostas (3).

As variáveis desta dimensão são compostas por indicadores de presença de fatores extrínsecos e intrínsecos baseados nos trabalhos de Deci et al. (1996), Cokley (2000), Pinder (2008) e Ertas (2015).

Em relação ao Estilo de Gestão médio das dimensões, as organizações produtoras rurais apresentaram uma média de 3,74 e mediana de 3,68, com desvio padrão relativamente baixo quando comparado com os demais. Estes resultados indicam que, embora estejam um pouco acima do regular, ainda existe possibilidade de melhorias por meio de intervenções pontuais nas áreas das dimensões avaliadas.

No sistema de Likert (Likert, 1967; Likert \& Likert, 1976), as organizações participantes seriam classificadas no Sistema 3. Este sistema é caracterizado como consultivo, apresentando interação, comunicação e tomada de decisão com razoável participação dos funcionários. Embora ocorra a participação do pessoal envolvido, a decisão final ainda é realizada pelo gestor.

O próximo passo de melhoria das organizações seria alcançar o Sistema 4, no qual predomina a participação dos funcionários nas tomadas de decisão, é utilizado fortemente o trabalho em equipe e a comunicação é realizada de forma horizontal e vertical (Likert, 1967; Likert \& Likert, 1976). 
Considerando que as dimensões utilizadas e o Estilo de Gestão são preditores de resultados organizacionais positivos, conforme observado na elaboração das hipóteses, qualquer melhoria nos índices das dimensões, em tese, poderia melhorar os resultados organizacionais.

\subsection{Discussões a partir dos dados obtidos por meio do grupo focal}

Os resultados obtidos por meio da análise quantitativa foram sintetizados e apresentados para o grupo, em que foram incitados a emitir suas opiniões livremente, tendo como insights as questões apresentadas na seção anterior. Após a transcrição da reunião, foram elaborados 3 códigos que guiaram as análises: Características das propriedades rurais com 12 citações, Diferenças entre grupos de gestores com 10 citações e Fatores do Estilo de Gestão com 11 citações.

O primeiro ponto de discussão apresentado referente às características dos produtores rurais visitados e pesquisados pelos participantes do grupo focal, distribuídos nos pontos de discussão, está apresentado no Quadro 2.

Quadro 2. Pontos de discussão do código características das propriedades rurais.

\begin{tabular}{|c|c|c|}
\hline Código & Pontos de discussão & Síntese dos argumentos \\
\hline \multirow{5}{*}{$\begin{array}{l}\text { Características } \\
\text { das } \\
\text { propriedades }\end{array}$} & \multirow[b]{2}{*}{ Acesso à informação } & Informação correlacionada à tecnologia \\
\hline & & $\begin{array}{l}\text { Baixa prioridade no acesso da informação sobre } \\
\text { gestão }\end{array}$ \\
\hline & $\begin{array}{l}\text { Diferenças no Estilo de } \\
\text { Gestão }\end{array}$ & $\begin{array}{l}\text { Diferentes equipes de gestão apresentam diferentes } \\
\text { estilos de gestão }\end{array}$ \\
\hline & Características técnicas & Modelo de produção: Multicultura \\
\hline & $\begin{array}{l}\text { Relação entre tecnologia } \\
\text { e número de } \\
\text { funcionários }\end{array}$ & $\begin{array}{l}\text { Quanto mais tecnologia menor quantitativo de } \\
\text { funcionários. }\end{array}$ \\
\hline
\end{tabular}

Fonte: Dados da pesquisa, apoio software Atlas TI.

A primeira característica apresentada é referente ao acesso à informação. De acordo com os participantes, eles possuem muito acesso à informação, mas pouca expertise em lidar com elas. Neste ponto, as discussões mostraram uma relação direta entre a informação e a tecnologia: "Os grandes produtores, me parece que eles já atendem bem a questão tecnológica, o próximo desafio deles é a gestão mesmo (Participante 1)". "Vejo que os produtores têm muita resistência em utilizar esse tipo de informação de gestão, se não for dado agronômico ele resiste. Não sei se é por falta de conhecimento ou resistência mesmo (Participante 3)".

Esta ênfase da informação estabelece ligação prioritária com a tecnologia de produção, deixando os aspectos relacionados à gestão em planos secundários.

Em relação à alteração do Estilo de Gestão quando alterada a equipe de gestão, sobre estes dois aspectos, os participantes do grupo focal discutiram características conceituais do construto Estilo de Gestão embasadas por Likert (1967, 1971), Likert \& Likert (1976), Tull \& Albaum (1971), Guerreiro (1989), Abramson et al. (1993), Culpan \& Kucukemiroglu (1993), Pereira \& Santos (2001), Blanco Jiménez et al. (2009), Petry \& Nascimento (2009), Rafiq \& Chishti (2011), Gomes et al. (2013) e Gupta et al. (2014), tratando o Estilo de Gestão como a manifestação das características de administrar de um gestor ou coletivo de gestores de uma determina organização (segundo aspecto), utilizando-se dos seus processos cognitivos que refletem todas suas atividades (primeiro aspecto).

A produção multicultura e a integração entre as diversas culturas também foi marcada como uma das características, como nas observações dos participantes: "Porque a gente tem $1^{a}, 2^{a}$ e $3^{a}$ cultura, você tem integração, você tem às vezes pecuária como atividade principal e também área de plantação (Participante 3)". "O cara que planta soja, ou ele planta milho, ou ele planta algodão, ou ele planta feijão na segunda safra, na realidade ele não planta soja, ele planta grãos, ou pastagem (Participante 1 )". 
Outro aspecto marcado do código características gerais está relacionado à tecnologia e quantitativo de pessoal, destacando uma correlação inversa: "Quanto maior a tecnologia, menor o número de funcionários (Participante 3)".

Considerando que a produção está muito ligada à utilização da tecnologia, com base nas informações dos participantes do grupo focal, pode-se correlacionar o número de funcionários dos participantes da pesquisa (survey) com a o volume de produção, resultando em uma correlação significante e positiva de $14 \%$ entre estes fatores, não confirmando as informações fornecidas pelos participantes do grupo focal.

O próximo código da análise de conteúdo versa sobre as diferenças na forma como as propriedades são geridas pelos proprietários e pelos gestores contratados. Os pontos desta discussão estão representados no Quadro 3.

Quadro 3. Pontos de discussão do código diferenças entre proprietários e gestores contratados.

\begin{tabular}{|c|c|c|}
\hline \multirow{2}{*}{ Código } & Pontos de discussão & \multicolumn{2}{c|}{$\begin{array}{c}\text { Síntese dos argumentos } \\
\text { Diferenças entre } \\
\text { pecuaristas e agricultores } \\
\text { entre } \\
\text { proprietários e } \\
\text { gestores } \\
\text { contratados }\end{array}$} & $\begin{array}{c}\text { Diferenças entre } \\
\text { proprietáncios e gestores } \\
\text { funcionários }\end{array}$ & $\begin{array}{c}\text { Existência da diferença no Estilo de Gestão dos } \\
\text { agricultores e dos pecuaristas }\end{array}$ \\
\cline { 2 - 3 } & $\begin{array}{c}\text { proprietários e dos gestores funcionários } \\
\text { Diferenças entre regiões }\end{array}$ & $\begin{array}{c}\text { Existência da diferença no Estilo de Gestão das } \\
\text { organizações da região sul das demais e entre os } \\
\text { Estados. }\end{array}$ \\
\hline
\end{tabular}

Fonte: Dados da pesquisa, apoio software Atlas TI.

Conforme apresentado por Morris \& Pavett (1992), Culpan \& Kucukemiroglu (1993), Beechler \& Yang (1994), Bakhtari (1995), Glunk et al. (1996), Pheng \& Leong (2001), Blanco Jiménez et al. (2009), características entre grupos de gestores de nações diferentes são manifestadas de forma díspares, embora, em algum dos casos, os gestores atuassem nas mesmas organizações. Ou mesmo como na pesquisa de Gupta et al. (2014), apresentando diferenças entre o Estilo de Gestão de homens e mulheres.

Neste ponto, os participantes elucidaram informações mostrando que existem diferenças entre a forma de gerir dos proprietários e funcionários.

A gente que trabalha aqui nessa região que é bem agrícola, a gente conhece um pouco da pecuária e um pouco da lavoura. [...] Porque o perfil do produtor que trabalha com agricultura é gigantescamente diferente de quem trabalha com pecuária. Que esse cara da agricultura consegue ir para a pecuária, mas o cara da pecuária não consegue ir para a agricultura. A não ser que ele precise muito (Participante 2 ).

A questão foi abordada por um dos participantes apresentando um estilo de gestão superior dos agricultores quando comparados com os pecuaristas, evidenciando que os fatores que moldam o estilo de gestão dos agricultores tendem a ser mais complexos.

Especificando essas diferenças, o participante mostrou que as principais diferenças são relacionadas ao uso das tecnologias: "Porque se eu pegar o cara dos grãos que é um cara altamente tecnológico, as respostas vão mudar ou não, permanecem parecidas. Agora se colocar da pecuária, já apresenta outro cenário (Participante 2)".

Os dados quantitativos da pesquisa sobre o Estilo de Gestão utilizados para contribuir com essa afirmação mostraram que os gestores cuja única atividade se caracteriza por agrícola apresentaram um score médio de 3,83 na escala (42 gestores), os estritamente pecuaristas apresentaram média de 3,84 (25 gestores).

Pelos dados buscou-se avaliar outros dois tipos de propriedades, as que realizam ambas as atividades de agricultura e pecuária, apresentando médias de 3,62 na escala (22 gestores), os que desenvolvem atividades de agricultura e outras atividades que não somente agricultura e pecuária mostraram média de 3,3 (4 gestores) e os que apresentaram atividades de pecuária e outras apresentaram média de 3,47 no Estilo de Gestão (4 gestores). 
Estas análises estatísticas não corroboram a informação dada pelos participantes do grupo focal, uma vez que as médias entre os pecuaristas e agricultores são muito próximas. Ainda neste ponto, foi discutida a questão de a tecnologia determinar o Estilo de Gestão.

Outro ponto abordado nas discussões foi pertinente à diferença entre o Estilo de Gestão dos proprietários das empresas rurais e dos funcionários que exercem atividades de gestão. As diferenças apresentadas pelos participantes foram: "Os proprietários são mais conservadores (Participante 1)". O gestor (proprietário) tem uma percepção extremamente positiva da empresa, poucas falhas, poucos defeitos e quando você vai no operacional, eles detonam a empresa (Participante 3)".

Diante disso, os participantes sugeriram realizar uma análise dos dados estratificada por proprietários $(n=43)$ e gestores funcionários $(n=58)$. Os resultados coletados pela técnica survey mostraram que os proprietários apresentaram uma média do Estilo de Gestão de 3,58 e os gestores funcionários apresentaram média de 3,87, diferindo das discussões realizadas pelo grupo focal.

O último ponto de discussão do código diferenças entre os grupos de gestores corresponde a diferenças entre as regiões brasileiras: "Como você (autor) tem participantes do sul, são áreas menores, acho interessante segmentar por Estado também (Participante 3)".

Neste aspecto, existem diferenças no Estilo de Gestão entre as regiões, predominando uma melhor avaliação dos demais Estados (média=3,92, $n=58$ ) em relação à média dos Estados da região sul do país (média 3,49, n=42), correspondendo às informações discutidas na reunião do grupo focal.

Em relação ao código fatores do Estilo de Gestão, os pontos abordados estão dispostos no Quadro 4.

Quadro 4. Pontos de discussão do código fatores do Estilo de Gestão.

\begin{tabular}{|c|c|c|}
\hline Código & Pontos de discussão & Síntese dos argumentos \\
\hline \multirow{6}{*}{$\begin{array}{l}\text { Fatores do } \\
\text { Estilo de } \\
\text { Gestão }\end{array}$} & Fator informação & $\begin{array}{l}\text { Possibilidade de maior ênfase do tema } \\
\text { informação nos fatores }\end{array}$ \\
\hline & Fator atualização & $\begin{array}{l}\text { Possibilidade de maior ênfase do tema } \\
\text { atualização nos fatores }\end{array}$ \\
\hline & Fator gestão de pessoas & $\begin{array}{l}\text { Possibilidade de inclusão do fator gestão de } \\
\text { pessoas }\end{array}$ \\
\hline & Fator Cultura Organizacional & $\begin{array}{c}\text { Possibilidade de inclusão do fator cultura } \\
\text { organizacional }\end{array}$ \\
\hline & Fator Tecnologia & Possibilidade de inclusão do fator tecnologia \\
\hline & Fator sucessão familiar & $\begin{array}{l}\text { Possibilidade de inclusão do fator sucessão } \\
\text { familiar. }\end{array}$ \\
\hline
\end{tabular}

Fonte: Dados da pesquisa, apoio software Atlas TI.

Os pontos oriundos do código fatores do Estilo de Gestão foram elucidados a partir de uma questão apresentada aos participantes do grupo focal:

"Além destes fatores utilizados na pesquisa, na opinião dos senhores, quais outros fatores são essenciais para caracterizar o Estilo de Gestão?"

Os fatores que surgiram durante as discussões, apresentados no Quadro 4, são eles: Informação, Atualização, Gestão de Pessoas, Cultura Organizacional, Tecnologia e Sucessão Familiar, conforme descrito nas falas.

Eu acho que reflete, porém, eu senti ausência, talvez ela esteja implícita do item informação, porque ele tem implicações na decisão, tem implicações no planejamento, e nas pesquisas que eu fiz, os produtores o tempo todo eles respiram isso. "Ah, eu preciso de informação disso, daquilo, daquele outro, desde mercado, capital, tecnologia". E com base nisso eles vão gerenciando o caos no bom sentido, que é as suas propriedades. Não sei se tem alguma coisa que tá implícita, mas no meu ponto de vista deveria ter contemplado. Informação e atualização, porque no contexto que eles dizem que tudo muda, cada safra é uma tese pra eles, [...] e que 
faz a diferença em mantê-los na atividade, o que eu tenho a sugerir é colocar atualização e informação (Participante 1).

Tem outras variáveis aí, a variável sucessão familiar, volto a falar de cultura organizacional, é relevante para saber como ela é gerida, tecnologia, técnicas de plantio como o plantio direto que antes não se falava, não se tirava a máxima produtividade do solo. Hoje você tem essas variáveis aí que precisam ser levadas em consideração (Participante 3).

"A tecnologia, informação, mercado, o conhecimento, a experiência que o produtor tem no ramo que ele está" (Participante 4).

"A variável cultura organizacional aparece em algum momento aqui ou não? Porque isso pode estar ligado se a pessoa tem informação, que é importante para entrar na variável de conhecimento" (Participante 3).

Embora os fatores Informação e Atualização não tenham sido sugeridos para serem tratados como um fator, mas compondo os outros fatores com maior ênfase, o tema informação foi considerado na elaboração dos fatores liderança, decisão, controle, planejamento e comunicação. Em relação ao tema atualização, nenhuma das sentenças utilizadas no instrumento quantitativo possui características que atendam ao tema.

Contudo, os pesquisadores Likert \& Likert (1976), Beechler \& Yang (1994), Glunk et al. (1996) consideraram Atualização como parte do Estilo de Gestão utilizando sentenças que mensurem o construto Treinamento.

Além disso, faz parte do próximo tema sugerido, Gestão de Pessoas, que por vez está em parte contido nos fatores Liderança e Motivação do instrumento de avaliação. Contudo, não atende a todos os componentes que o tema exige tais como os utilizados por Demo et al. (2014): Recrutamento e Seleção, Envolvimento, Treinamento, desenvolvimento e educação, Condições de trabalho, Avaliação de desempenho e competências e Remuneração e Recompensas.

O tema seguinte, Tecnologia, foi discutido de forma recorrente durante toda a reunião. É considerada pelos participantes como uma característica muito presente nas organizações produtoras rurais. Este tema não foi considerado na elaboração das sentenças do instrumento de avaliação do Estilo de Gestão.

O tema Cultura Organizacional mencionado por um dos participantes também foi abordado por Zeffane (1994) como componente do Estilo de Gestão, respondendo por questões ligadas mais aos aspectos organizacionais do que aos individuais. Embora não faça parte inteiramente do instrumento de avaliação da parte quantitativa desta Tese, alguns dos componentes desta temática estão presentes, tais como: Normas e regras sendo parte das sentenças do fator Controle e Valores Organizacionais no fator Planejamento.

Por fim, o tema sucessão familiar também foi apresentado por um dos participantes do grupo, o qual não foi contemplado na elaboração do instrumento quantitativo, como também nenhuma das pesquisas analisadas na revisão da literatura apontou este fator como componente do Estilo de Gestão.

Assim como o tema Tecnologia, Sucessão Familiar e os demais, podem ser fatores relevantes para avaliar o Estilo de Gestão específico dos produtores rurais.

Os pontos de discussão apresentados pelos participantes da pesquisa puderam ser confrontados com os dados coletados por meio da técnica de levantamento (survey), sendo alguns pontos corroborados pelos participantes como os (1) fatores utilizados na composição do Estilo de Gestão, (2) as diferenças entre os Estilos de Gestão de diferentes grupos que compuseram a amostra da pesquisa e outro que não foi corroborado como a diferença entre o Estilo de Gestão dos pecuaristas e agricultores.

Além disso, elucidaram novas possibilidades de inclusão de fatores que podem compor o Estilo de Gestão como Tecnologia, Sucessão Familiar, Cultura Organizacional, Atualização (ou treinamento), Gestão de Pessoas e Informação, como sugestão para futuras pesquisas. 


\section{Considerações Finais}

A presente pesquisa teve como objetivo discutir a gestão dos produtores rurais brasileiros com base no Estilo de Gestão. Muito embora a gestão de organizações seja uma discussão extremamente complexa, esta pesquisa avançou na elucidação de partes desta complexidade, além de oportunamente avaliar um objeto de pesquisa muito singular para a economia brasileira, com vistas à exploração de suas características de gestão.

As poucas pesquisas que trataram do Estilo de Gestão como um construto das ciências da administração mostraram várias formas de avaliar a gestão organizacional, caracterizada como complexa e multidimensional. Neste contexto, foi possível responder à questão problema desta pesquisa: Como é o Estilo de Gestão dos Produtores Rurais Brasileiros?

Portanto, o Estilo de Gestão dos Produtores Rurais Brasileiros, na amostra pesquisada, se caracteriza pelos elevados níveis de aplicação das atividades de liderança, decisão, controle, motivação e tecnologia, menor relevância nos aspectos planejamento e comunicação que parecem estar preteridas e carentes de atenção dos gestores. Este resultado está balizado pelos resultados obtidos por meio do instrumento quantitativo de avaliação e ponderado pelas argumentações obtidas durante a reunião do grupo focal, uma vez que a gestão é considerada a nova fronteira para melhorar o desempenho das propriedades rurais.

Comparando os resultados obtidos com as pesquisas analisadas na revisão da literatura, esta pesquisa permitiu avaliar a partir das percepções dos gestores, resgatando o objetivo inicial da pesquisa de Likert \& Likert (1976). Além disso, permitiu avaliar o Estilo de Gestão com maior profundidade utilizando o grupo focal para verificar o que existe além do que a pesquisa de levantamento atingiu.

Como sugestões para pesquisas futuras, indica-se incorporar os fatores Informação, Atualização (ou treinamento), Gestão de Pessoas, Cultura Organizacional, Tecnologia e Sucessão Familiar na avaliação do Estilo de Gestão, conforme sugerido pelos participantes do grupo focal na avaliação do Estilo de Gestão

Diante da apresentação destes resultados e suas contribuições, espera-se que se tenha avançado nas discussões e estimulado novos debates sobre o Estilo de Gestão de organizações do agronegócio no País.

\section{Agradecimentos}

O presente trabalho foi realizado com apoio da Coordenação de Aperfeiçoamento de Pessoal de Nível Superior - Brasil (CAPES) - Código de Financiamento 001; e Apoio da Universidade Federal de Mato Grosso do Sul - UFMS.

\section{Referências}

Abramson, N. R., Lane, H. W., Nagai, H., \& Takagi, H. (1993). A comparison of Canadian and Japanese cognitive styles: implications for management interaction. Journal of International Business Studies, 24(3), 575-588.

Alhassan, M. A., Ghazali, Z., \& Isha, A. S. N. (2014). Communication and temperament in the choice of conflict management styles among plant turnaround maintenance employees in PETRONAS petrochemical companies in malaysia: a conceptual framework. Global Business and Management Research. International Journal (Toronto, Ont.), 6(4), 262-270.

Armstrong, C. M., Niinimäki, K., Lang, C., \& Kujala, S. (2016). A use-oriented clothing economy? preliminary affirmation for sustainable clothing consumption alternatives. Sustainable Development 24(1), 18-31.

Avolio, B. J., Waldman, D. A., \& Yammarino, F. J. (1991). Leading in the 1990s: the four i's of transformational leadership. Journal of European Industrial Training, 15(4), 9-16.

Bakhtari, H. (1995). Cultural effects on management style: a comparative study of american and middle eastern management styles. International Studies of Management \& Organization, 25(3), 97-109.

Banks, G. C., Pollack, J. M., Bochantin, J. E., Kirkman, B. L., Whelpley, C. E., \& O’Boyle, E. H. (2016). Management's science-practice gap: a grand challenge for all stakeholders. Academy of Management Journal, 59(6), 2205-2231.

Bardin, L. (1977). Análise de conteúdo. Lisboa: Edições 70. 
Bass, B. M., \& Avolio, B. J. (1997). Full range leadership development: manual for the multifactor leadership questionnaire. California: MindGarden.

Beechler, S., \& Yang, J. Z. (1994). The transfer of japanese-style management to american subsidiaries: contingencies, constraints, and competencies. Journal of International Business Studies, 25(3), 467492.

Blanco Jiménez, M., Fasci, M. A., \& Valdez, J. (2009). A comparison of management style for mexican firms in Mexico and the Unit States. International Journal of Business, 14(3), 251-263.

Bojanic, A. H. (2017). The rapid agricultural development of Brazil in the last 20 years. EuroChoices, 16(1), 5-10.

Brasil. Ministério da Agricultura, Pecuária e Abastecimento. (2010). Agronegócio brasileiro em números. Brasília: MAPA. Recuperado em 16 de abril de 2015, de http://www.agricultura.gov.br/comunicacao/publicacoes

Brasil. Ministério da Agricultura, Pecuária e Abastecimento. (2016). Balança Comercial - Série Histórica (1989-2015). Brasília: MAPA. Recuperado em 19 de abril de 2016, de http://www.agricultura.gov.br/

Campos, E. A. R., Stefano, S. R., \& Andrade, S. M. (2015). Cultura organizacinal no contexto de Políticas e Práticas de Gestão de Pessoas. Reuna, 20(3), 65-82.

Cardoso, O. O. (2006). Comunicação empresarial versus comunicação organizacional: novos desafios teóricos. Revista de Administração Pública, 40(6), 1123-44.

Centro de Estudos Avançados em Economia Aplicada - CEPEA. (2013). Relatório PIBagro - Brasil. Piracicaba: ESALQ/USP. Recuperado em 2 de maio de 2014, de http://cepea.esalq.usp.br/pib/

Child, J., Elbanna, S., \& Rodrigues, S. (2010). The political aspects of strategic decision making. In P. C. Nutt \& D. C. Wilson (Eds.), Handbook of decision making (pp. 105-137). West Sussex: Wiley.

Cokley, K. (2000). Examining the validity of the Academic Motivation Scale by comparing scale construction to self-determination theory. Psychological Reports, 86(2), 560-564.

Costa Neto, P. L. O. (1977). Estatística (264 p.). São Paulo: Edgard Blücher,

Culpan, R., \& Kucukemiroglu, O. (1993). A comparison of U.S. and japanese management styles and unit effectiveness. Management International Review, 33(1), 27-42.

Cummings, S., \& Daellenbach, U. (2009). A guide to the future of strategy: the history of long range planning. Long Range Planning, 42(2), 234-263.

Dantas, R. A. S. (2007). Adaptação cultural e validação do questionário do senso de coerência Antonovsky em uma amostra de pacientes cardíacos brasileiros (Tese de doutorado). Escola de Enfermagem de Ribeirão Preto, Universidade de São Paulo, Ribeirão Preto.

Davis, J. H., \& Goldeberg, R. (1957). A concept of agribusiness. Boston: Havard University.

De Koning, J. C., Crul, M. R. M., Wever, R., \& Brezet, J. C. (2015). Sustainable consumption in Vietnam: an explorative study among the urban middle class. International Journal of Consumer Studies, 39(6), 608-618.

Deci, E. L., Ryan, R. M., \& Williams, G. C. (1996). Need satisfaction and the self-regulation of learning. Learning and Individual Differences, 8(3), 165-183.

Demo, G., Neiva, E. R., Nunes, I., \& Rozzett, K. (2014). Políticas e práticas de recursos humanos. In M. M. M. Siqueira (Org.), Novas medidas do comportamento organizacional: ferramentas de diagnóstico e gestão. Porto Alegre: Artmed.

Dufour-Poirier, M., \& Laroche, M. (2015). Revitalising young workers' union participation: a comparative analysis of two organisations in Quebec (Canada). Industrial Relations Journal, 46(5/6), 418-433.

Eisenhardt, K. M., \& Zbaracki, M. J. (1992). Strategic decision making. Strategic Management Journal, 13(No. esp.), 17-37.

Ertas, N. (2015). Turnover intentions and work motivations of millennial employees in federal service. Public Personnel Management, 44(3), 401-423.

Fischmann, A. A., \& Almeida, M. I. R. (2009). Planejamento estratégico na prática. São Paulo: Atlas.

Fowler Junior, F. (2011). Pesquisa de levantamento. Porto Alegre: Penso.

Franco, S. M., \& Leão, A. L. M. S. (2013). Codificando/decodificando a comunicação organizacional: uma contribuição dos estudos culturais. Revista Pensamento Contemporâneo em Administração, 7(1), 32 49.

Gasques, J. G. (2017). Sources of growth in brazilian agriculture: total factor productivity. EuroChoices (Uckfield), 16(1), 24-25. 
Ghasabeh, M. S., Soosay, C., \& Reaiche, C. (2015). The emerging role of transformational leadership. Journal of Developing Areas, 49(6), 459-467.

Gil, A. C. (2008). Métodos e técnicas de pesquisa social (6. ed.). São Paulo: Atlas.

Glunk, U., Wilderom, C., \& Ogilvie, R. (1996). Finding the key to German-style management. International Studies of Management \& Organization, 26(3), 93-108.

Gomes, C. M., Schuch Junior, V. F., Madruga, L. R. G., \& Kneipp, J. M. (2013). Estilos de gestão: um estudo em pequenas e médias empresas. Pretexto, 14(3), 99-113.

Goodman, D., Sorj, B., \& Wilkinson, J. (2008). Da lavoura às biotecnologias: agricultura e indústria no sistema internacional. Rio de Janeiro: Centro Edelstein de Pesquisas Sociais.

Grabner, I., \& Moers, F. (2013). Management control as a system or a package? Conceptual and empirical issues. Accounting, Organizations and Society, 38(6), 407-419.

Graffin, S., Boivie, S., \& Carpenter, M. (2013). Examining CEO successions and the role of heuristics in early-stage CEO evaluation. Strategic Management Journal, (34), 384-403.

Guerreiro, R. (1989). Modelo conceitual do sistema de informação de gestão econômica: uma contribuição à teoria da comunicação da contabilidade (Tese de doutorado). Faculdade de Economia, Administração e Contabilidade da Universidade de São Paulo, São Paulo.

Guimarães, T. B., Pordeus, I. A., \& Campos, É. S. A. (Org.). (2010). Estado para resultados: avanços no monitoramento e avaliação da gestão pública em Minas Gerais. Belo Horizonte: Editora UFMG.

Gupta, V. K., Javadian, G., \& Jalili, N. (2014). Role of entrepreneur gender and management style in influencing perceptions and behaviors of new recruits: evidence from the Islamic Rebublic of Iran. Journal of International Entrepreneurship, 12, 85-109.

Hair Junior, J. F., Black, W. C., Babin, B. J., \& Anderson, R. E. (2010). Multivariate data analysis (7th ed.). Upper Saddle River: Pearson Prentice Hall.

Hall, S. (2003). Codificação/Decodificação. In S. Hall. Da diáspora: identidades e mediações culturais. Belo Horizonte: Editora UFMG.

Hared, B. A., Abdullah, Z., \& Huque, S. M. R. (2013). Gestão de Sistemas de Controle: uma revisão da literatura e um quadro teórico para futuras pesquisas. European Journal of Business and Management, 5(26), 1-13.

Hartono, S., \& Sobari, A. (2016). Coopetition, cluster externalities and company performances: formation for competitiveness of the wood and rattan furniture industry. International Journal of Organizational Innovation, 9(2), 271-286.

Heredia, B., Palmeira, M., \& Leite, S. P. (2010). Sociedade e economia do "Agronegócio" no Brasil. Revista Brasileira de Ciências Sociais, 25(74), 159-196.

Huang, X. (2009). Strategic decision making in Chinese SMEs. Chinese Management Studies, 3(2), 87-101.

Hubbard, H., Alvim, A. M., \& Garrod, G. (2017). Brazilian agriculture as a Global Player. EuroChoices, 16(1), 3-4.

lizuka, K., Takei, Y., Nagase, R., \& Suematsu, C. (2014). Satisfaction structure of the implementation effect of Enterprise Resource Planning (ERP): an analysis from the management style perspective of japanese firms. International Journal of Business and Information, 9(3), 273-310.

Instituto Brasileiro de Geografia e Estatística - IBGE. (2010). Classificação Nacional de Atividades Econômicas - CNAE. Recuperado em 27 de junho de 2016, de cnae.ibge.gov.br

Instituto Matogrossense de Economia e Agropecuária - IMEA. (2014a). Agronegócio no Brasil e em Mato Grosso. Recuperado em 2 de maio de 2014, de http://www.imea.com.br/projetos.php

Instituto Matogrossense de Economia e Agropecuária - IMEA. (2014b). Manual prático da conjuntura econômica. In Workshop Jornalismo Agropecuário. Recuperado em 27 de junho de 2014, de http://www.imea.com.br/upload/pdf/arquivos/Apresentacao_Conjuntura_Economica_Imea_parte_1 .pdf

King, R. P., Boehlje, M., Cook, M. L., \& Sonka, S. T. (2010). Agribusiness economics and management. American Journal of Agricultural Economics, 92(2), 554-570.

Kistruck, G. M., Lount, R. B., Smith, B. R., Bergman, B. J., \& Moss, T. W. (2016). Cooperation vs. competition: alternative goal structures for motivating groups in a resource scarce environment. Academy of Management Journal, 59(4), 1174-1198.

Lam, P. (2016). Chinese culture and coaching in Hong Kong. International Journal of Evidence Based Coaching and Mentoring, 14(1), 57-73.

Likert, R. (1967). The human organization: its management and value. Nova lorque: MacGraw-Hill.

Likert, R. (1971). Novos padrões de administração. Nova lorque: MacGraw-Hill. 
Likert, R., \& Likert, J. G. (1976). New ways of managing conflict. Nova lorque: MacGraw-Hill.

Lindell, M., \& Arvonen, J. (1996). The nordic management style in a european context. International Studies of Management \& Organization, 26(3), 73-91.

Lomi, A., Conaldi, G., \& Tonellato, M. (2012). Organized anarchies and the network dynamics of decision opportunities in an open source software project. In A. Lomi \&J. R. Harrison (Eds.), The garbage can model of organizational choice: looking forward at forty (Research in the Sociology of Organizations, Vol. 36, pp. 363397). Bingley: Emerald Group Publishing Limited.

Maguire, S., \& Hardy, C. (2009). Discourse and deinstitutionalization: the decline of DDT. Academy of Management Journal, 52(1), 148-178.

Marchiori, M. (2009). A relação Comunicação-Organização: uma reflexão sobre seus processos e práticas. In Anais do $3^{\circ}$ Congresso da Abrapcorp. São Paulo: USP. Recuperado em 3 de março de 2016, de http://www.abrapcorp.org.br/anais2009/pdf/gt2_marlene_marchiori.pdf

Mariño-Arévalo, A. (2014). Las relaciones de poder y la comunicación en las organizaciones: una fuente de cambio. AD-minister, 24, 119-141.

Martínez-Aires, M. D., Rubio Gámez, M. C., \& Gibb, A. (2016). The impact of occupational health and safety regulations on prevention through design in construction projects: perspectives from Spain and the United Kingdom. Work (Reading, Mass.), 53(1), 181-191.

Merchant, K., \& Van Der Stede, W. (2007). Management control systems: performance measurement, evaluation and incentives (2. ed.). Harlow: Prentice-Hall.

Mintzberg, H. (2008). Criando organizações eficazes: estruturas em cinco configurações (2. ed.). São Paulo: Atlas.

Moreddu, C., Contini, E., \& Ávila, F. (2017). Challenges for the Brazilian Agricultural Innovation System. EuroChoices (Uckfield), 16(1), 26-31.

Morris, T., \& Pavett, C. M. (1992). Management style and productivity in two cultures. Journal of International Business Studies, 23(1), 169-179.

Mukherji, A., Mukherji, J., Wright, P., \& Hasan, S. (2009). The impact of entrepreneurial orientation, strategic capabilities, management style, and growth focus on firm performance. Competition Forum, 7(1), 84-90.

Oliveira, A. A., Aquino, M. C., \& Silva, W. T. (2016). O endomarketing como estratégia na gestão de pessoas: influências de clima e cultura nas organizações. E3 Revista de Economia, Empresas e Empreendedores na CPLP, 2(1), 1-18.

Oliveira, D. P. R. (2014). Planejamento estratégico: conceitos, metodologia e práticas (32. ed.). São Paulo: Atlas.

Pasquali, L. (2010). Testes referentes a construto: teoria e modelo de construção. In L. Pasquali (Org.), Instrumentação psicológica: fundamentos e práticas (p. 165-168). Porto Alegre: Artmed.

Pelegrino, F. M. (2009). Adaptação cultural e validação do instrumento Duke Anticoagulation Satisfaction Scale (DASS): versão para brasileiros em uso de anticoagulação oral (Dissertação de mestrado). Escola de Enfermagem de Ribeirão Preto, Universidade de São Paulo, Ribeirão Preto.

Pereira, C. A. (2011). Gestão econômica. In C. Parisi \& E. Megliorini (Org.), Contabilidade gerencial (2. ed.). São Paulo: Atlas.

Pereira, M. I., \& Santos, S. A. (2001). Modelo de gestão: uma análise conceitual. São Paulo: Pioneira Thomson Learning.

Perreira, T., \& Berta, W. (2016). Using focus groups to modify the Workplace Affective Commitment Multidimensional Questionnaire (WACMQ) for use in health care. Journal of Health and Human Services Administration, 39(3), 407-424.

Petry, L. I., \& Nascimento, A. M. (2009). Um estudo sobre o modelo de gestão e o processo sucessório em empresas familiares. Revista Contabilidade \& Finanças, 20(49), 109-125.

Pheng, L. S., \& Leong, C. H. Y. (2001). Asian management style versus Western management theories - A Singapore case study in construction project management. Journal of Managerial Psychology, 16(2), 127-141.

Pinder, C. C. (2008). Work motivation in organizational behavior (2nd. ed.). Nova lorque: Psychology Press.

Poister, T. H. (2010). The future of strategic planning in the public sector: linking strategic management and performance. Public Administration Review, 70(No. esp.), 246-254.

Poon, P. S., Evangelista, F. U., \& Albaum, G. (2005). A comparative study of the management styles of marketing managers in Australia and the People's Republic of China. International Marketing Review, 22(1), 34-47.

Porpino, G., Parente, J., \& Wansink, B. (2015). Food waste paradox: antecedents of food disposal in low income households. International Journal of Consumer Studies, 39(6), 619-629.

Rafiq, M., \& Chishti, S. H. (2011). Relationship between management style and organizational performance: a case study from Pakistan. International Journal of Academic Research, 3(5), 290-293. 
Ramos, S. C., Takahashi, A. R. W., \& Roglio, K. D. (2015). Análise da Produção Nacional sobre Processo Decisório no Período de 2004-2014. Contextus - Revista Contemporânea de Economia e Gestão, 13(3), 156-184.

Rivers, L., Dempsey, T., Mitchell, J., \& Gibbs, C. (2015). Environmental regulation and enforcement: structures, processes and the use of data for fraud detection. Journal of Environmental Assessment Policy \& Management, 17(4), 1550033-1-1550033-29.

Robbins, S. P., \& Decenzo, D. A. (2004). Fundamentos de administração: conceitos essenciais e aplicações (4. ed.). São Paulo: Prentice Hall.

Schumann, C., Bowman, N. D., \& Schultheiss, D. (2016). The quality of video games: subjective quality assessments as predictors of self-reported presence in first-person shooter and role-playing games. Journal of Broadcasting \& Electronic Media, 60(4), 547-566.

Serra, J. P. (2007). Manual de teoria da comunicação. Covilhã: Universidade da Beira Interior.

Severino, A. F. (2010). Força do clima: o papel do consenso intragrupo (Dissertação de mestrado). Universidade de Brasília, Brasília.

Shadare, O. A. (2011). Management style and demographic factors as predictors of managerial efficiency in work organizations in Nigeria. International Business \& Economics Research Journal, 10(7), 85-93.

Silva, J. G. (1996). A nova dinâmica da agricultura brasileira. Campinas: Unicamp-Instituto de Economia.

Simon, H. A. (1945). Administrative behavior: a study of decision-making processes in administrative organization. Nova lorque: Macmillan Co.

Simons, R. (1995). Levers of control: how managers use innovative control systems to drive strategic renewal. Harvard Business School Press: Boston.

Sousa, J. P. (2006). Elementos de teoria e pesquisa da comunicação e dos media (2. ed.). Porto: Porto.

Souza, C. C. (2010). Os estudos culturais ontem e hoje: a codificação/decodificação de Hall aplicado ao hiperconsumidor pós-moderno. In Anais do $11^{\circ}$ Congresso de Ciências da Comunicação na Região Sul. Novo Hamburgo: Feevale.

Souza, S. C., \& Marinho, S. V. (2014). Planejamento estratégico baseado no Balanced Scorecard: um estudo de caso aplicado a uma pequena empresa de segurança. Revista Gestão \& Planejamento, 15(2), 213-237.

Spaho, K. (2013). Organizational communication and conflict management. Management, 18(1), 103-118.

Srivastava, M., \& Banerjee, P. (2016). Understanding Gen Y. Journal of Management Research, 16(3), 148-164.

Tull, D. S., \& Albaum, G. (1971). Decision processes and the valuation of information. Oregon Business Review, $30(4), 1-6$

Vaitsman, J., Rodrigues, R. W. S., \& Paes-Sousa, R. (2006). O sistema de avaliação e monitoramento das políticas e programas sociais: a experiência do Ministério do Desenvolvimento Social e Combate à Fome do Brasil. Brasília: Unesco.

Virgílio, A. P. S. (2017). Agricultura moderna brasileira e aprofundamento da dependência a partir dos anos 1990: 0 caso do agronegócio da soja (Dissertação de mestrado). Universidade Federal da Bahia, Salvador.

Vitória, F., Almeida, L. S., \& Primi, R. (2006). Unidimensionalidade em testes psicológicos: conceito, estratégias e dificuldades na sua avaliação. Revista de Psicologia da Vetor Editora, 7(1), 1-7.

Wanyonyi, N., Frantz, J., \& Saidi, H. (2015). The effect of a knowledge-based ergonomic intervention amongst administrators at Aga Khan University Hospital, Nairobi. Work (Reading, Mass.), 52(4), 843854.

Zakrison, T. L., Cabezas, P., Valle, E., Kornfeld, J., Muntaner, C., \& Soklaridis, S. (2015). The perceived consequences of gold mining in postwar el salvador: a qualitative study. American Journal of Public Health, 105(11), 2382-2387.

Zeffane, R. (1994). Patterns of organizational commitment and perceived management style: a comparison of public and private sector employees. Human Relations, 47(8), 977-1010.

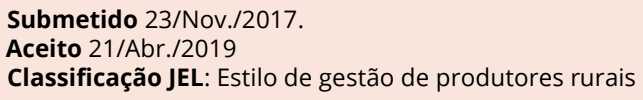

